\title{
Density- and age-dependent reproduction partially compensates culling efforts of invasive non-native American mink
}

Article

Accepted Version

Melero, Y., Robinson, E. and Lambin, X. (2015) Density- and age-dependent reproduction partially compensates culling efforts of invasive non-native American mink. Biological Invasions, 17 (9). pp. 2645-2657. ISSN 1573-1464 doi: https://doi.org/10.1007/s10530-015-0902-7 Available at https://centaur.reading.ac.uk/83110/

It is advisable to refer to the publisher's version if you intend to cite from the work. See Guidance on citing.

To link to this article DOI: http://dx.doi.org/10.1007/s10530-015-0902-7

Publisher: Spinger

All outputs in CentAUR are protected by Intellectual Property Rights law, including copyright law. Copyright and IPR is retained by the creators or other copyright holders. Terms and conditions for use of this material are defined in the End User Agreement.

www.reading.ac.uk/centaur 
Central Archive at the University of Reading

Reading's research outputs online 
1 Density- and age-dependent reproduction partially compensates culling efforts of invasive non-

2 native American mink

3

$4 \quad$ Yolanda Melero $^{1,2}$ Ella Robinson ${ }^{1}$ and Xavier Lambin ${ }^{1}$

5 (1) School of Biological Sciences, University of Aberdeen. Aberdeen AB24 2TZ, UK.

6 (2) CREAF (Center for Ecological Research and Forestry Applications), Autonomous University of

7 Barcelona, E-08193 Bellaterra, Spain

8

$9 \quad(1,2)$ Yolanda Melero: y.melero@abdn.ac.uk; Present address: y.melero@creaf.uab.es

10 (1) Ella Robinson: ella.robinson.10@aberdeen.ac.uk

11 (1) Xavier Lambin (corresponding author): x.lambin@abdn.ac.uk; phone: +44(0)1224 273259

12

13 


\section{Abstract}

15 Management strategies of wildlife species must pay due regard to density dependent changes in vital rates. Knowledge of density dependent relationships is sparse for most species but such knowledge ought to inform adaptive management. Using data from a large-scale, six years of control effort of the invasive non-native American mink (Neovison vison) in Scotland, we analysed density dependent changes in reproduction as revealed by placental scar counts in culled females. Control strongly reduced mink density but it varied substantially over time and space, reflecting variation in when control was initiated in each river section. We used hurdle statistical models to simultaneously relate the probability of conception, litter size and female age to prevailing mink density in river sections where the female was culled. Both the probability of conceiving a litter (average 0.81 ) and litter size (average 5.52 pups) increased as the density of females, but not males, declined. In addition, there was a senescent decline in both components of fecundity, which given culling of mink and subsequent reinvasions, resulted in a younger population, adding further to density dependent compensation in fecundity. There was no evidence of depensation, even at the lowest density. The predicted combined impact of changes in density and age structure could lead to an increase in fecundity of up to 2.1 pups per female occupying or reinvading the controlled area. Control strategies must be sufficiently adaptable and robust in order to overcome this compensation and suppress densities of mink and other invasive mammals.

33 Keywords Density dependence; compensation; senescence; fecundity; introduced species 
Density dependence demographic processes underpin the sustainable harvest of wildlife species.

Indeed, a reduction in population density may lead to density dependent compensation (i.e. per capita growth rate increases as population density decreases). as a consequence of abundant per capita resource availability at low density. In some circumstances however, individuals living in low and sparsely populated areas may experience depensation or Allee effects (i.e. per capita growth rate decreases as the density decreases) which may reduce their fitness (Courchamp et al., 2008). Strong Allee effects may even give rise to inverse (or positive) density dependence, which in principle could result in a minimum threshold population size below which the population growth rate becomes negative and extinction is expected (Gregory \& Courchamp 2010).

While changes in per-capita resource availability typically underpin patterns of density dependent variation in the population growth rate, the specific limiting resources driving the changes are rarely identified (Sinclair and Pech, 1996). In addition to inducing density dependent changes in vital rates, elevated mortality through harvesting may truncate age and size structures. This truncation typically leads to more young animals in harvested populations with a subsequent impact on vital rates that are not density dependent per se (e.g. Robinson et al. 2008). Studies of animal populations occurring at low densities are notoriously difficult to perform and hence relatively scarce. As a result, much uncertainty remains on the relative contributions of compensatory and depensatory processes in shaping per capita growth rate in low-density populations for species with different social systems or reproductive physiologies. Indeed, evidence of depensation is controversial and largely restricted to failures of cooperative processes in social species across taxa (Gregory et al., 2010; Kramer et al., 2009). In this respect, control campaigns of nefarious introduced non-native species (INNS hereafter) designed to reduce the abundance of target species to very low values provide useful, quasi-experimental conditions to study density dependent processes. While eradication of INNS is the management objective on islands not subjected to immigration, reducing INNS to densities where their ecological impact on native communities becomes benign is widely practiced in "mainland areas" where ultimate eradication is a distant goal 
controlled through sustained trapping rather than through one-off poisoning campaigns in areas where the use of toxins is inappropriate (e.g. Bonesi \& Palazón 2007). Management strategies involving either large numbers of "citizen conservationists" (e.g. Bryce et al. 2011) or substantial injection of resources (Ebbert and Byrd, 2011; Harding et al., 2001) have successfully reduced INNS to low densities over large areas. In most cases, the species involved are territorial, solitary, highly mobile and fecund mammals with relatively low survival (e.g. mongooses, feral cats Felis catus and American mink Neovison vison). To date, consideration of the roles of compensatory and depensatory processes as well as demographic stochasticity in INNS biology has been largely restricted to the early establishment stage of invasive species spread (e.g. Sax et al., 2007). Yet the same processes may also contribute to invasive species' response to reduction in population density following control programs, and as such, are of both fundamental and applied interest. However, empirical evidence of compensatory and depensatory mechanisms in wildlife species is still scarce and mostly represented by compensatory dispersal from the outer perimeter of controlled area (e.g. Englund 1970), or by juvenile dispersers (e.g. Berry et al. 2013). Little is known about how changes in in situ reproduction by individuals who either escaped control or immigrated might contribute to compensation or depensation in the persisting low density population. Specifically, the relative contribution of variation in per capita resource availability and mate finding to variation in the reproductive output is scarcely known for most pest species.

Here we use data from a large-scale participatory INNS control effort, during which densities of a small carnivore were reduced over a very large area, to determine whether compensatory and depensatory processes affecting reproduction countered or facilitated control.

\section{Study Species}

The American mink (mink hereafter) is a widely distributed mammalian INNS and the focus of much control effort worldwide (see Dunstone, 1993; Genovesi et al., 2012; Santulli et al., 2014). Mink is a highly mobile, solitary, territorial species occupying linear territories arranged along waterways. Mating takes place in March-April with males abandoning their territories and becoming nomadic in 
search of mating opportunities during the rut (Dunstone, 1993). Female mink mate with, and can be fertilised by, multiple males per mating season (superfecundation). They continue ovulating after successfully mating (superfoetation) and have embryonic diapauses which synchronise embryonic development (Dunstone, 1993; Thom et al., 2004). The combination of these characteristics results in the production of a single annual litter of typically $4-5$ offspring (up to 14), frequently of different paternities (Yamaguchi et al., 2004). Kits are born in April-May and can fend for themselves by late August. Juveniles leave the maternal territory and disperse from October when approximately 5 months old and seek a vacant territory in which they typically settle by the end of the year. Once settled, mink do not abandon their territories except for males during mating. Juveniles mature sexually when 8-10 months old (Dunstone, 1993; Melero and Palazón, 2011).

Based on knowledge of mink social system and reproductive physiology and the assumption that food availability constrains body condition and fecundity, we predicted that female fecundity would rise with decreasing mink density. We assumed that territory size would become less constrained by the presence of conspecifics and that immigrants would be able to settle in the best quality territories. We thus expected that females would be able to attain higher body condition and hence conceive larger litters. We further envisaged that as mink density declined to very low values, the number of mating events per oestrus would decline. Thus we predicted that the relationship between litter size and mink density would be non-linear, with a decline below a threshold reflecting a reduction in mating frequency and an increasing fraction of females failing to conceive at very low male density.

\section{Material and methods}

The project

Carcasses of mink culled as part of the Cairngorm Water vole Conservation Project (2006-2009; Bryce et al. 2011) and its successor the Scottish Mink Initiative (2009-present) were collected in an area centred around the Cairngorms National Park $\left(57^{\circ} 0^{\prime} \mathrm{N} 3^{\circ} 3^{\prime} \mathrm{W}\right)$ in northern Scotland. The culling 
area extended to $10,000 \mathrm{~km}^{2}$ by 2009 and gradually expanded to cover more than $20,000 \mathrm{~km}^{2}$ in 2013 .

118 Both projects used mink rafts (details in Reynolds et al., 2010), which were deployed at regular

119 intervals along waterways and operated more or less continuously by volunteers, with support from one to four project officers. A cage trap was placed on the raft only when a mink was detected and left in place for a few days or until a mink was caught (38\% of detections resulted in capture in 20062009; Beirne, 2011). The spatial coverage of the projects expanded over time but in a non-systematic manner. This variation was largely a consequence of the temporal and spatial variation in project funding (details in Bryce et al. 2011). The projects were funded through a succession of awards, each of one to three years duration, and there were also some interim periods with minimal funding. This led to variation in levels of staffing and hence variation in the level of mink detection effort by volunteers, the intensity of mink raft coverage and in the collection of data. There was a marked temporary decline in late 2009 in particular.

Mink sampling

Carcasses of culled mink were frozen upon collection from volunteers. Uteri were then extracted and refrozen until subsequent examination. Embryos and placental scars in the uteri were counted to estimate litter size. In mink, as in other mammals with a zonary endotheliochorial or discoid hemochorial type of placenta (Burton et al., 1994), the uteri is left with one placental scar per implanted embryo that heals within 10-11 months of parturition. The scars become visible and can be counted when the uteri is stained following the protocol described by Fournier-Chambrillon et al. (2010). A single observer (YM) counted the scars twice in quick succession and gave a subjective confidence score to each count, as some scars were difficult to discern. Mink age was estimated in two steps. First an X-ray image of canines was taken and individuals were classed as younger or older than 10 months based on the appearance of dental pulp (Helldin, 1997). Those judged to be older than 10 months were further aged using tooth cementum analyses which was performed by Matson

142 Laboratory LLC (MT, USA). Throughout, kits were assumed to be born in May and females were assumed to be able to conceive from 10 months old. 
Derived variables

The expansion of the mink control projects and the collection of mink carcasses took place in a heterogeneous physical landscape with overlaid heterogeneity both in the timing of comprehensive mink control being first implemented and in the number of conspecifics known to persist when each focal mink was caught (see Bryce et al. 2011). In order to reflect this heterogeneity, we divided the project area into 21 river sections (average length of waterways: $153 \mathrm{~km}, \mathrm{SD}=26$ ) with a consistent control history. Each section was assigned a "year since first controlled". In order to estimate the degree of saturation of mink territories (i.e. the relative mink density post control) in each river section at a given time, we estimated the maximum number of territories per section. To do so, we first buffered all capture locations of settled adult mink in all years by $2 \mathrm{~km}$ along rivers. Next, we merged the buffers to describe areas which were ever settled by mink and divided the total occupied length of waterway by $2 \mathrm{~km}$ territories assuming no-overlap (Birks and Linn, 1982; Dunstone and Birks, 1987). Relative mink densities were then taken to be the ratio of the number of occupied territories per year and section, relative to the maximum number of territories estimated under the above assumptions (Fig 1a). Even though saturation of river section with territories was an empirical cumulative measure, 15 of 21 sections had reached the maximum number of territories by the second year of control, meaning that no new mink territories were discovered after the second year (Appendix: Fig. 1).

Relative mink densities were calculated per year for (a) adult conspecifics of both sexes as a measure of competition for territories; (b) adult females as a measure of intersexual competition for territories and mating opportunities; (c) adult males reflecting competition for territories after the rut; and (d) mating males as a measure of mating opportunities. Mating males were all the potential mating partners present during the mating season of year $t$. They were defined as adult males captured from the beginning of March to the end of the calendar year (year t hereafter) and the individual male mink captured from January to the end of February the next year (year $t+1$ ) if $>21$ months old (Fig $1 b)$. 
171 To quantify the mating opportunities that females experienced, while taking into account the mobility

172 of males during the rut, we calculated an index of connectivity between all relevant adult males and

173 each focal oestrus female. The connectivity index $S^{m m}$ was the sum of the number of mating males

174 weighed by a negative exponential function of the Euclidean distance (in $\mathrm{m}$ ) between the focal

175 females $i$ and the location of capture of each male $d_{i j}$. Keeping in mind that the capture location of

176 males may not reflect their location at the time of mating, $S^{m m}$ should only be considered as an index

177 of the number of mating opportunities. $S^{m m}$ was expressed as: $S_{i}^{m m}=\sum e^{\left(-d_{i j} / d^{\prime}\right)}$ where $S^{m m}$ increases with the number of adult male mink in a female's neighbourhood, and decreases with the distance following a negative exponential function shaped by the parameter $d^{\prime}$ (also expressed as $\alpha=$ $1 / d^{\prime}$ ). The parameter $d^{\prime}$ should reflect the mobility and mate finding ability of mink and was estimated from the data using profile likelihood. The global models (see statistical analysis below), with probability of conception and litter size as response variables, were iteratively fitted to the data using values of $S^{m m}$ estimated for a range of values of $d^{\prime}(1000-40000)$. The most likely value of $d^{\prime}$ was taken as that which yielded the model with the highest log-likelihood. When $d^{\prime}$ is large, connectivity becomes the total number of males un-weighted by distance $\left(d_{i j}\right)$. All covariates, including connectivity, were standardized by subtracting the mean and dividing by two standard deviations. Because the best models did not include connectivity between females and males (see section 3.2), the relative density of mating males was not calculated by section but rather per year over the entire project area, as the ratio of mating males' territories relative to the maximum number of territories that could have been accommodated in that year.

Hypotheses testing and statistical analyses

When testing our predictions on density dependence in mink fecundity, we modelled pregnancy status (whether the female had conceived a litter or not) and litter size (the number of placental scars) in relation to four measures of mink density (a-d, testing first for co-linearity), including the connectivity index to mating males. To investigate the influence of the control history of each river section on pregnancy rate and litter size, we also tested the time (in years) elapsed from "year since first 
controlled". In all models, we also considered female age (in months) at conception (setting April as

199 the month of conception), and the month of capture to account for possible changes in the

200 detectability of placental scars after parturition.

201 In all analyses, we first sought evidence of non-linear relationships between mink fecundity and

202 indices of mink density or the month of capture, using generalised additive mixed models (GAMM),

203

204

205

206

207

208

209

210

211

212

213

214

215

216

217

218

219

220

221

222

223

224

225 with year as a random variable. Wherever smoothing terms were non-significant, we used hurdle models, also called mixture models, because the zeros are modelled as arising from two different processes: the binomial process (of conceiving) and the count process (of the number of embryos implanted) fitted as a truncated Poisson distribution (Zuur et al 2009). Confidence in the measurement of placental scars based on two counts was included in all relevant models by weighing each observation according to:

$W_{i}=\left(1-\left(L S_{i 1}-L S_{i 2}\right) / 5\right) x R_{i 1} / 100$, where the weight $(W)$ of each count of litter size $i$ is a function of the difference between the two counts ( $i 1$ and $i 2)$ divided by five to avoid zeros and multiplying by the confidence score $R_{i l}$ scaled $0-1$. For each analysis, a global model was defined containing all mentioned covariates and model selection was conducted by sequentially dropping terms based on AIC (Table 1 in Supplementary material). Only additive effects were considered. Model averaging and estimates weighting across the most likely models $(\triangle \mathrm{AIC}<1)$ were used to incorporate model uncertainty in the parameter estimates using the R package MuMIn (Bartoń, 2014). Analyses were carried out in R using packages gamm4, pscl and nlme (Jackman, 2012; Pinheiro et al , 2014; Wood, 2014).

\section{Results}

A total of 979 mink were captured of which 387 were female, 452 were male and 140 were of unrecorded sex. Amongst females, 238 were $>10$ months old, hence potentially reproductive. Only seven females were culled when pregnant. The repeated placental scar counts were identical for $97 \%$ of instances. Overall, $81 \%$ of the females conceived, with an average litter size of 5.52 ( $\mathrm{SD}=2.89$; Fig. 2a, b). There were four instances of litters of one pup, in different river sections and years. One 
22611 months old female at parturition had an apparent litter size of 12 (based on placental scars) in the

227 first year of control of a river section. Female age at conception varied between years from 11 to 59

228

229

230

231

233

234

235

236

237

238

239

240

241

242 months $(\sim 5$ years) old with an average of 16.76 months $(\mathrm{SD}=10.86)$ (Fig. $2 \mathrm{c}, \mathrm{d})$. The proportion of females judged to have conceived declined with the calendar month of capture, from all females in January to $81 \%$ in December. A similar pattern was evident for the estimated litter size which varied from six to three pups (Table 1, Appendix: Fig. 2 and 3). These patterns persisted when 22 litters from females culled in January-March with above average size were excluded from the analyses.

\section{Population relative density and female age}

The relative density of conspecifics per section varied widely between river sections and fluctuated over time. There was a consistent decline in density when time since control was instigated, with a project-wide reduction of $80 \%$ after six years of control. Relative density decreased to less than $20 \%$ of the estimated maximum in six sections, and to $50 \%$ in eight other sections during the first year of control. Relative mink density in the remaining seven sections increased in the first but decreased in the second year of control (Fig. 3a, b). Overall, 57\% of culled females inhabited river sections with relative densities lower than 20 and $6.7 \%$ females at relative densities lower than 10 and $5 \%$ of the local maximum respectively.

None of our indices of relative density were significantly or strongly correlated to each other (all p-values $>0.1$ ), with the relative density of conspecifics and females having the highest Pearson correlation $r_{p}=0.23$.

Culling lead to an increasingly young population, with the percentage of females older than two years declining from an average of $38 \%$ in the first two years of control to $23 \%$ in the fifth year and $0 \%$ in the sixth when all culled females were yearlings (Fig. 3b).

Modelling mink fecundity: density dependence and senescence 
251

252

253

254

255

256

257

258

259

260

261

262

263

264

265

266

267

268

269

270

271

272

273

274

275

276

277

Our prediction of density dependence was supported by the best models of fecundity. The part of the hurdle model relating to the probability that a female conceived included negative relationships with the relative density of females, as well as with female age at conception and month of capture. The litter size part of the model also included the above covariates, as well as relative densities of males and mating males (Appendix: Table 1). Our prediction of a non-linear effect of density on fecundity was not supported since no smoothing terms were deemed to be significant for any of the GAMM models, providing no evidence for non-linearity in the influence of any of the density indices indicative of depensation. The best models relating conception probability and litter size in relation to mating males did not include connectivity between females and males ( $\max d^{\prime} \Delta \log$ likelihood $=0.15$; Appendix: Table 1 and Fig. 4). Throughout, model selection was unaffected by weighing observation according to repeatability $(w)$.

Probability of conception decreased monotonically with an increase in the relative density of females and the age of the focal female. Using all other covariates within their real range of values, the hurdle model predicted conception probabilities ranging from 0.92 (95\% CI 0.86-0.96) at the minimum recorded relative densities of females $(<2 \%)$ to $0.82(0.51-0.93)$ at the maximum relative densities (84\%). Predicted probability of conception declined in relation to female age from 0.86 (0.73-0.94) in yearling females $(n=127)$ to $0.55(0.23-0.85)$ in the oldest females in the fifth breeding year (59 months old, n=2) (Table 1 and Fig. 4a, b). The effects of both covariates on the probability of conception were equivalent in strength (coefficients of standardised covariates: -0.369 and -0.351 respectively, Table 1).

Litter size also declined with increasing relative density of females and with female age. Predicted litter size declined from 5.6 pups (interval confidence 4.5-6.4) at the minimum recorded densities to 3.8 pups (2.9-4.8) at maximum densities, while within the female age at parturition litter size declined from 4.9 (4.8-5) in yearlings to 3.1 (2-4.8) pups in the eldest females (Fig. 4c, d). Despite being part of the best model selected based on AIC scores, the impact of relative density of males and of mating males on litter size had an effect size close to zero, with a litter size of 4.9 pups seemingly invariant with male densities. Thus despite being selected in the best models (Fig. 5), 
model parameters (either weighted or not) offer no evidence to support the notion that the potential for multiple mating leads to higher litter size.

\section{Compensation in fecundity and mink control efforts}

Both population density and the average female age declined over the course of the management project (Fig. 2d and Fig. 3c, d), and both influenced mink fecundity through the conception rate and litter size components of fecundity. To illustrate the maximum model-predicted magnitude of their combined impact, we selected two of the 21 project sections, which had constant coverage since the start of the project and current low densities (Fig. 3). We used the observed range of their local values for female density and age while holding all other covariates at their median value. Litter size was predicted to have increased by 1.5 fold, from 3.67 (95\% CI 2.42-5.26) pups in the first year of control to 5.49 (95\% CI 4.56-6.61; Fig. 2b) after six years of control. The probability of conception was predicted to have increased from 0.78 (95\% CI 0.79-0.93) to 0.91 (95\% CI 0.83-0.96). When considering the range of density and female age observed in all river sections, the model predicted a combined increase of 2.1 pups per litter (95\% CI 1.0-3.3). No such pattern is evident in the raw mink fecundity data (Fig. 2b) owing to the fluctuations of asynchrony between river sections with different control histories in their relative female density and age (Fig. 3d).

\section{Discussion}

We found evidence of a density dependent response in both conception rates and female fecundity to the culling-induced decline of an invasive American mink population, with a negative relationship between fecundity and relative density of females but not with the other indices of density we evaluated. We found no evidence of depensation. In addition, we found evidence of reproductive senescence, with a decrease in both the probability of conceiving and litter size with age. Mink culling lead to a younger, more fecund mink population which ought to have contributed to an overall compensation effect over the course of the management project. 
Sustained mink culling over multiple river catchments led to a substantial reduction in the relative density of females with increasing number of years of control. The compensatory increases in the proportion of females conceiving and in litter sizes must have partly counteracted control efforts. Clearly the overall compensatory response was insufficient to negate the effects of culling since density declined continuously within the years of control. Compensation effects are commonly observed in the wild as a consequence of relaxation of competition at low density (e.g. Boyce et al., 1999; Fryxell et al., 1999, 2001). We derived a pragmatic estimate of mink relative density by comparing the number of settled mink in a given year with the potential number that could have settled by combining information on the likely location of breeding territories over several years. On that basis, mink densities in eight out of 21 river sections were never saturated, as in these areas the maximum density in any year never reached the inferred maximum number of potential territories, which were inferred from the capture locations of females (Fig. 3). This could indicate that female mink were caught in, and possibly occupied, sub-optimal habitats in some years, denoting scope for compensation.

While keeping in mind the caveats inherent to a non-experimental study like this one, the statistical influence of female density on fecundity points towards female-biased intersexual competition for territories occurring in American mink. While most females do conceive each year, the increase in both conception rate and litter size suggest females attain better physiological condition at low density. If conception and litter size were constrained by nutritional status, preferential settlement of females in the best, most productive territories, might have contributed to the pattern we observed.

We postulated that features of female mink reproductive physiology might give rise to a positive relationship between the number of males a female mated with and litter size. Despite a substantial fraction of the culled females originating from river sections with severely depleted male mink, our measures of the availability of males in spring provided no strong evidence of mating limitation or of 
any impact on litter size. Indeed, the relative density of females was the most important predictor of litter size and, together with age, was one of the two most important predictors of the probability of conception. We expected that mink, being solitary animals, would have experienced a reduction in the probability of conception because of inefficient mate finding at the lowest densities. However, assuming that the proxy for density of mating males we used reflects mating opportunities, mate availability only had a marginal effect on litter size and none on the probability of conception. We considered separately the densities of females and conspecifics of either sex. Only female relative density contributed to explaining variation in mink reproduction. On that basis, we find no evidence for inter-sexual competition for territories such that the evidence is more consistent with the notion of female territoriality.

The combined effect of age and female density reduction led to a predicted compensatory increase in mink fecundity of up to 2.1 additional pups per surviving or immigrating female. Keeping all other traits constant and using a simple two stage, female-only matrix model with adult and yearling survival set at $0.5 \%$ (Melero, 2007), the resulting increase in overall fecundity would increase the annual population multiplication rate from 1.19 to 1.39 , a difference of $26 \%$. We know of no reliable estimates of population growth rate for American mink, but our simple model approximations are in line with estimates for similar sized mustelids such as the American marten (Martes americana), feral ferret (Mustela putorius) and black footed ferret (Mustela nigripes), which can range between 1.31.35 (Barlow and Norbury, 2001; Fryxell et al., 1999; Grenier et al., 2007). While this illustrates the potency of an increase in fecundity for a short-lived species like mink, it is of course possible that other traits such as post weaning juvenile survival could also be density-dependent and that the overall compensation to culling might be greater still.

\section{Senescence in mink fecundity}

In addition to density dependence, mink fecundity was affected by senescence, with the youngest breeding females having the largest litters and a higher pregnancy rate, resulting in higher fecundity. 
360 Reproductive senescence has been detected in ungulates (e.g. McElligott et al., 2002; Jolles, 2007)

361 and rodents (reviewed in Nussey et al., 2013) but evidence is scarce for carnivores, being restricted to

362 long-lived species such as badgers (Dugdale et al., 2011), lions (Packer et al., 1988) and bears

363 (Schwartz et al., 2003). In a short-lived species with young age at primiparity such as mink,

364 senescence is expected to occur earlier than in long-lived ones (Bradley and Safran, 2014) but little

365 evidence is available. Litter size was reported to be independent of age in fur-farmed mink over the

366 first three years of age (Koivula et al., 2010). Thus to the best of our knowledge, this is the first time

367 that senescence in fecundity is detected in free ranging mink, and to our knowledge, in a short-lived

368 carnivore.

369

370

Consequences and applications

371

Despite a predicted increase in mink fecundity due to density dependence and changes in age structure combined with senescence, the current management regime that entails ongoing mink detection followed by trapping has succeeded in controlling invasive mink density over a large area. The model prediction of an increase in fecundity was evident amongst females that re-invaded those river sections in which mink control has been sustained over multiple years, with severely depressed density. No such increase was discernible over the whole project area because the project expanded in an asynchronous manner, with trapping in river sections beginning in different years. Our analyses stress the importance of precluding recolonisation of low density areas by juveniles born in adjacent areas and of early detection of immigrants before they express their potentially high fecundity.

Our analyses also uncovered a noticeable project area-wide increase in mink density in 2010, coinciding with the decline of staff and vigilance in late 2009. Fortunately a key feature of the mink management protocol implemented in this project, i.e. the fact that mink trapping effort is not constant but adjusted locally in response to the detection of mink footprints on mink detection rafts, overcame 
there is a need to continue intensive detection and response to trapping in the event of any localised mink reinvasion to counteract the density dependent increase in mink fecundity that putatively could lead to the production of large litters of young, potentially dispersive mink by surviving breeding females. While the management regime instigated by the management projects caters for this eventuality (Bryce et al 2011), maintaining vigilance by volunteers operating mink rafts when mink are scarce is an ongoing challenge.

\section{Author contributions}

Y.M and X.L. designed the study. Y.M. and E.R. carried out the laboratory uteri staining procedures. Y.M. analysed the data and with X.L. wrote the manuscript.

\section{Acknowledgements}

Y.M. was funded by a Marie Curie FP7-PEOPLE-2011-IEF 300288-Project Depensation and XL by NERC grant NE/J01396X/1. We thank the Scottish Mink Initiative, staff, funders and multiple mink volunteers for the continued effort, samples and data, Alex Douglas and Matthew K. Oliver for statistical advice. We also thank MSc. William Morgan, MSc. Ewan Mchenry and Dr. Emma Sheehy for their English grammar and spelling correction.

\section{References}

Barlow, N., Norbury, G., 2001. A simple model for ferret population dynamics and control in semiarid New Zealand habitats. Wildl. Res. 28, 87-94. doi:10.1071/WR99090

Bartoń, K., 2014. MuMIn: Multi-model inference. R package version 1.10.5.

Barun, A., Hanson, C.C., Campbell, K.J., Simberloff1, D., 2011. A review of small Indian mongoose management and eradications on islands, in: Veitch, C.R., Clout, M.N., Towns, D.R. (Eds.), Island Invasives: Eradication and Management. UICN, Gland, Switzerland], pp. 17-25.

Beirne, C., 2011. Novel use of mark-recapture framework to study volunteer retention probabilities within an invasive non-native species management project reveals vocational and temporal trends. University of Aberdeen. 
Berkeley, S.A., Hixon, M.A., Larson, R.J., Love, M.S., 2004. Fisheries Sustainability via Protection of Age Structure and Spatial Distribution of Fish Populations. Fisheries 29, 23-32. doi:10.1577/1548-8446(2004)29[23:FSVPOA]2.0.CO;2

Berry, O., Tatler, J., Hamilton, N., Hilmer, S., Hitchen, Y., Algar, D., 2013. Slow recruitment in a red-fox population following poison baiting: a non-invasive mark-recapture analysis. Wildl. Res. 40, 615. doi:10.1071/WR13073

Birks, J.D.S., Linn, I.J., 1982. Studies of home range of the feral mink, Mustela vison. Symp. Zool. Soc. London 49, 231-257.

Bomford, M., O'Brien, P., 1995. Eradication or Control for Vertebrate Pests? Wildl. Soc. Bull. 23, 249-255.

Bonesi, L., Palazón, S., 2007. The American mink in Europe: Status, impacts, and control. Biol. Conserv. 134, 470-483. doi:10.1016/j.biocon.2006.09.006

Boyce, M.S., Sinclair, A.R.E., White, G.C., 1999. Seasonal Compensation of Predation and Harvesting. Oikos 87, 419-426.

Bradley, R.J., Safran, R.J., 2014. Conceptual Revision and Synthesis of Proximate Factors Associated with Age-Related Improvement in Reproduction. Ethology 120, 411-426. doi:10.1111/eth.12220

Bryce, R., Oliver, M.K., Davies, L., Gray, H., Urquhart, J., Lambin, X., 2011. Turning back the tide of American mink invasion at an unprecedented scale through community participation and adaptive management. Biol. Conserv. 144, 575-583. doi:10.1016/j.biocon.2010.10.013

Burton, G., Kaufmann, P., Huppertz, B., 1994. Anatomy and genesis of the placenta, in: Knobil, E., Neill, J. (Eds.), The Physiology of Reproduction. Raven Press, New York, pp. 441-484.

Courchamp, F., Berec, L., Gascoigne, J., 2008. Allee effects in ecology and conservation. Oxford University Press, Oxford.

Dugdale, H.L., Pope, L.C., Newman, C., Macdonald, D.W., Burke, T., 2011. Age-specific breeding success in a wild mammalian population: selection, constraint, restraint and senescence. Mol. Ecol. 20, 3261-74. doi:10.1111/j.1365-294X.2011.05167.x

Dunstone, N., 1993. The Mink. T and A D Poyserd Ltd, London.

Dunstone, N., Birks, J.D.S., 1987. The feeding ecology of mink (Mustela vison) in coastal habitat. J. Zool. 212, 69-83.

Ebbert, S.., Byrd, G.., 2011. Eradication of Invasive species to restore natural biological diversity on Alaska Maritime National Wildlife Refuge, in: Veitch, C.R., Clout, M.N. (Eds.), Turning the Tide: The Eradication of Invasive Species. UICN, Gland, Switzerland, pp. 102-109.

Enders, R.K., 1952. Reproduction in the mink (Mustela Vison). Proc. Am. Philos. Soc. 96, 691-755.

Englund, J., 1970. Some aspects of reproduction and mortality rates in Swedish foxes (Vulpes vulpes) 1961-63 and 1966-69. Viltrevy 8, 1-82. 
Fournier-Chambrillon, C., Bifolchi, A., Mazzola-Rossi, E., Sourice, S., Albaret, M., Bray, Y., Ceña, J.-C., Maya, F.U., Agraffel, T., Fournier, P., 2010. Reliability of stained placental scar counts in farmed American mink and application to free-ranging mustelids. J. Mammal. 91, 818-826. doi:10.1644/09-MAMM-A-297.1

Fryxell, J., Falls, J., Falls, E., Brooks, R., 1999. Density dependence, prey dependence, and population dynamics of martens in Ontario. Ecology 80, 1311-1321.

Fryxell, J.M., Falls, B., Falls, E.A., Brooks, R.J., Dix, L., Strickland, M., 2001. Harvest dynamics of mustelid carnivores in Ontario, Canada. Wildlife Biol. 7, 169-177.

Gaillard, J.-M., Festa-Bianchet, M., Yoccoz, N.G., Loison, A., Toigo, C., 2000. Temporal variation in fitness components and population dynamics of large hervibores. Annu. Rev. Ecol. Syst. 31, 367-393. doi:10.1146/annurev.ecolsys.31.1.367

Genovesi, P., Carnevali, L., Alonzi, A., Scalera, R., 2012. Alien mammals in Europe: updated numbers and trends, and assessment of the effects on biodiversity. Integr. Zool. 7, 247-253. doi:10.1111/j.1749-4877.2012.00309.x

Gregory, S.D., Bradshaw, C.J.A., Brook, B.W., Courchamp, F., 2010. Limited evidence for the demographic Allee effect from numerous species across taxa. Ecology 91, 2151-2161. doi:10.1890/09-1128.1

Gregory, S.D., Courchamp, F., 2010. Safety in numbers: extinction arising from predator-driven Allee effects. J. Anim. Ecol. 79, 511-4. doi:10.1111/j.1365-2656.2010.01676.x

Grenier, M.B., McDonald, D.B., Buskirk, S.W., 2007. Rapid Population Growth of a Critically Endangered Carnivore. Science (80-. ). 317, 779.

Harding, E.K., Doak, D.F., Albertson, J.D., 2001. Evaluating the Effectiveness of Predator Control: the Non-Native Red Fox as a Case Study. Conserv. Biol. 15, 1114-1122. doi:10.1046/j.15231739.2001.0150041114.x

Helldin, J.-O., 1997. Age determination of Eurasian Pine martens by radiographs of teeth in situ. Wildl. Soc. Bull. 25, 83-88.

Jackman, S., 2012. pscl: Political Science Computational Laboratory, Stanford University. R package version 1.04.4.

Jolles, A.E., 2007. Population biology of African buffalo (Syncerus caffer) at Hluhluwe-iMfolozi Park, South Africa. Afr. J. Ecol. 45, 398-406. doi:10.1111/j.1365-2028.2006.00726.x

Jones, O.R., Gaillard, J.-M., Tuljapurkar, S., Alho, J.S., Armitage, K.B., Becker, P.H., Bize, P., Brommer, J., Charmantier, A., Charpentier, M., Clutton-Brock, T., Dobson, F.S., FestaBianchet, M., Gustafsson, L., Jensen, H., Jones, C.G., Lillandt, B.-G., McCleery, R., Merilä, J., Neuhaus, P., Nicoll, M.A.C., Norris, K., Oli, M.K., Pemberton, J., Pietiäinen, H., Ringsby, T.H., Roulin, A., Saether, B.-E., Setchell, J.M., Sheldon, B.C., Thompson, P.M., Weimerskirch, H., Jean Wickings, E., Coulson, T., 2008. Senescence rates are determined by ranking on the fastslow life-history continuum. Ecol. Lett. 11, 664-73. doi:10.1111/j.1461-0248.2008.01187.x

Koivula, M., Stranden, I., Mantysaari, E.A., 2010. Genetic and phenotypic parameters of age at first mating, litter size and animal size in Finnish mink. Animal 4, 183-188. 
Kramer, A.M., Dennis, B., Liebhold, A.M., Drake, J.M., 2009. The evidence for Allee effects. Popul. Ecol. 51, 341-354. doi:10.1007/s10144-009-0152-6

Lee, D.E., Tietje, W.D., 2005. Dusky-footed woodrat demography and prescribed fire in a california oak woodland. J. Wildl. Manage. 69, 1211-1220. doi:10.2193/0022541X(2005)069[1211:DWDAPF]2.0.CO;2

McElligott, A.G., Altwegg, R., Hayden, T.J., 2002. Age-specific survival and reproductive probabilities: evidence for senescence in male fallow deer (Dama dama). Proc. Biol. Sci. 269, 1129-37. doi:10.1098/rspb.2002.1993

Melero, Y., 2007. Study of the demographic parameters of the endangered European mink in the Spanish population. Anim. Biol. University of Barcelona, Barcelona.

Melero, Y., Palazón, S., 2011. Visón americano--Neovison vison (Schreber, 1777). In: En: Salvador, A., Cassinello, J. (Eds.). Encicl. Virtual los Vertebr. Españoles.

Melero, Y., Palazón, S., Bonesi, L., Gosàlbez, J., 2010. Relative abundance of culled and not culled American mink populations in northeast Spain and their potential distribution: are culling campaigns effective? Biol. Invasions 12, 3877-3885. doi:10.1007/s10530-010-9778-8

Nogales, M., Martin, A., Tershy, B.R., Donlan, C.J., Veitch, D., Puerta, N., Wood, B., Alonso, J., 2004. A Review of Feral Cat Eradication on Islands. Conserv. Biol. 18, 310-319. doi:10.1111/j.1523-1739.2004.00442.x

Nussey, D.H., Froy, H., Lemaitre, J.-F., Gaillard, J.-M., Austad, S.N., 2013. Senescence in natural populations of animals: widespread evidence and its implications for bio-gerontology. Ageing Res. Rev. 12, 214-25. doi:10.1016/j.arr.2012.07.004

Nussey, D.H., Kruuk, L.E.B., Donald, A., Fowlie, M., Clutton-Brock, T.H., 2006. The rate of senescence in maternal performance increases with early-life fecundity in red deer. Ecol. Lett. 9, 1342-50. doi:10.1111/j.1461-0248.2006.00989.x

Packer, C., Herbst, L., Pusey, A.E., Bygott, J.D., Hanby, J.P., Cairns, S.J., Mulder, M.B., 1988. Reproductive success of lions, in: Clutton-Brock, T.H. (Ed.), Reproductive Success: Studies of Individual Variation in Contrasting Breeding Systems. University of Chicago Press, Chicago, pp. 363-383.

Pinheiro J, Bates D, DebRoy S, S.D. and R.C.T., 2014. nlme: Linear and Nonlinear Mixed Effects Models. R package version 3.1-117.

Reynolds, J.C., Porteus, T. a., Richardson, S.M., Leigh, R.J., Short, M.J., 2010. Detectability of American Mink Using Rafts to Solicit Field Signs in a Population Control Context. J. Wildl. Manage. 74, 1601-1606. doi:10.2193/2009-209

Robinson, H.S., Wielgus, R.B., Cooley, H.S., Cooley, S.W., 2008. Sink populations in carnivore management: cougar demography and inmigration in a huntd population. Ecol. Appl. 18, 10281037. doi:10.1890/07-0352.1

Santulli, G., Palazón, S., Melero, Y., Gosálbez, J., Lambin, X., 2014. Multi-season occupancy analysis reveals large scale competitive exclusion of the critically endangered European mink by the invasive non-native American mink in Spain. Biol. Conserv. 176, 21-29. doi:10.1016/j.biocon.2014.05.002 
Sax, D.F., Stachowicz, J.J., Brown, J.H., Bruno, J.F., Dawson, M.N., Gaines, S.D., Grosberg, R.K., Hastings, A., Holt, R.D., Mayfield, M.M., O'Connor, M.I., Rice, W.R., 2007. Ecological and evolutionary insights from species invasions. Trends Ecol. Evol. 22, 465-471. doi:DOI: 10.1016/j.tree.2007.06.009

Schwartz, C.C., Keating, K.A., Reynolds, H.V., I., Barnes, V.G., J., Sellers, R.A., Swenson, J.E., Miller, S.D., McLellan, B.N., Keay, J., McCann, R., Gibeau, M., Wakkinen, W.F., Mace, R.D., Kasworm, W., Herrero, S., 2003. Reproductive maturation and senescence in the female brown bear. Ursus 14, 109-119.

Simberloff, D., Martin, J.-L., Genovesi, P., Maris, V., Wardle, D.A., Aronson, J., Courchamp, F., Galil, B., García-Berthou, E., Pascal, M., Pyšek, P., Sousa, R., Tabacchi, E., Vilà, M., 2013. Impacts of biological invasions: what's what and the way forward. Trends Ecol. Evol. 28, 58-66.

Simon Wood, F.S., 2014. gamm4: Generalized additive mixed models using mgcv and lme4. R package version 0.2-3.

Sinclair, A.R.E., Pech, R.P., 1996. Density Dependence, Stochasticity, Compensation and Predator Regulation. Oikos 75, 164-173.

Taylor, C.M., Hastings, A., 2005. Allee effects in biological invasions. Ecol. Lett. 8, 895-908. doi:10.1111/j.1461-0248.2005.00787.x

Thom, M.D., Johnson, D.D.P., Macdonald, D.W., 2004. The evolution and maintenance of delayed implantation in the mustelidae (Mammalia: Carnivora). Evolution (N. Y). 58, 175-183. doi:10.1111/j.0014-3820.2004.tb01584.x

Tobin, P.C., Berec, L., Liebhold, A.M., 2011. Exploiting Allee effects for managing biological invasions. Ecol. Lett. no-no. doi:10.1111/j.1461-0248.2011.01614.x

Walters, C., Kitchell, J.F., 2001. Cultivation/depensation effects on juvenile survival and recruitment: implications for the theory of fishing. Can. J. Fish. Aquat. Sci. 58, 39-50. doi:10.1139/f00-160

Yamaguchi, N., Sarno, R.J., Johnson, W.E., O'Brien, S.J., Macdonald, D.W., 2004. Multiple paternity an reproductive tactics of free-ranging American minks, Mustela vison. J. Mammal. 85, 432439.

Zuur, A., Ieno, E.N., Walker, N., Saveliev, A.A., Smith, G.M., 2009. Mixed Effects Models and Extensions in Ecology with R, XXII. ed. Springer, New York, USA. 
Table 1 Weighted parameter estimates of the influence of standardised covariates with their

561 associated standard errors for variables included in the best hurdle models for the Probability of

562 conception and the Litter size at parturition in relation to the relative density of females $\left(\mathrm{D}_{\mathrm{f}}\right)$, males

$563\left(\mathrm{D}_{\mathrm{m}}\right)$ and mating males $\left(\mathrm{D}_{\mathrm{mm}}\right)$, the female age at conception in months (Age) and the month of capture

564 (Mcapt).

\begin{tabular}{|c|c|c|c|c|}
\hline & Parameters & Estimate & SE & $\mathrm{Z}$ \\
\hline \multirow[t]{4}{*}{ Probability of conception } & Intercept & 3.607 & 1.005 & 3.586 \\
\hline & $\overline{D_{f}}$ & -0.369 & 0.237 & -1.568 \\
\hline & Age & -0.351 & 0.205 & -1.726 \\
\hline & Mcapt & -1.551 & 1.046 & -1.482 \\
\hline \multirow[t]{6}{*}{ Litter Size } & Intercept & 1.982 & 0.131 & 14.807 \\
\hline & $\mathrm{D}_{\mathrm{f}}$ & -0.067 & 0.103 & -1.181 \\
\hline & $\mathrm{D}_{\mathrm{m}}$ & -0.011 & 0.037 & -0.801 \\
\hline & $\mathrm{D}_{\mathrm{mm}}$ & -0.006 & 0.042 & -0.212 \\
\hline & Age & -0.028 & 0.048 & -0.543 \\
\hline & Mcapt & -0.263 & 0.159 & -1.41 \\
\hline
\end{tabular}

565

566

567 
570 Fig. 1 (a) Illustrative example of maximum number of mink territories; symbols represent capture

571 locations per years, circles are $2 \mathrm{~km}$ buffers and the colour code represents territories in different

572 years. In the example dark grey is 2007, grey 2008 and light grey 2009. (b) Illustrative example for

573 the definition of mating males, M1, M2 and M3 represent adult males captured in May 2009 (M2) and

574 February 2010 (M1 and M3). M1 and M2 were considered "mating males" of female F1 because they

575 were adult and still alive during rut 2009. M2 was not a potential mating male of F1 since it was born

576 after the rut 2009

577

578 Fig. 2 Violin plot for the litter size of females that conceived $(n=205)$ and age at parturition of all sampled females $(\mathrm{n}=238>10$ months old) in relation to the year and the number of years of control. Grey shapes show the density distribution of the y-axis covariate, grey circles stand for the median, black bars for the quartiles Q1 and Q3, straight black lines for the minimum and maximum values and black areas in (a) and (b) shows the density distribution of non-mated females.

Fig. 3 Relative density of mink per (a) year and (b) years of control and relative density of females per (c) year and (d) years of control. Solid black lines describe the project-wide relative density. Thin lines show relative densities per river section with black dots denoting the year of first control for each section, illustrating the gradual expansion of the project. Red and green lines notes the selected project sections with constant coverage since the start of the project and currently low densities to illustrate the maximum, model-predicted, magnitude of their combined impact.

591 Fig. 4 Observed (dot) and hurdle model derived predictions (lines) probability of conception in relation to (a) the relative density of females; (b) female age at conception; and litter size of females that conceived in relation to (c) the relative density of females, (d) female age at conception. Parameters were estimated using weighted estimates of the three top best models. Continuous line relates to the estimated fit setting all the other covariates as constant at their median value; dashed lines denote the $95 \%$ confidence intervals. 
598 Fig. 5 Observed (dot) and model predictions of the litter size of conceived females in relation to (a)

599 the relative density of males and (b) the relative density of mating males. Parameters were estimated

600 using weighted estimates of the three best models. Continuous line relates to the estimate fit setting all

601 the other covariates as constant at their median value dashed lines relate to the $95 \%$ confidence

602 intervals.

603

604

605

606

607 